\title{
Cardiopulmonary Resuscitation Complicated by Traumatic Hepatic Laceration: A Case Report and Review of Literature
}

\author{
Saptarshi Biswas ${ }^{\mathrm{a}, \mathrm{b}}$, Allisa Alpert ${ }^{\mathrm{a}}$, Marcus Lyon ${ }^{\mathrm{a}}$, Christoph R. Kaufmann ${ }^{\mathrm{a}}$
}

\begin{abstract}
We present an interesting case of a 63-year-old patient who was having lunch with friends in a restaurant when, suddenly, bystanders witnessed him "seize" for 1- minute, after which he became he had a 1-minute witnessed "seizure" followed by unresponsive and pulseless. Cardiopulmonary resuscitation (CPR) was initially performed by these bystanders at the scene, and then subsequently by EMS personnel, who achieved return of spontaneous circulation (ROSC) in the field. The patient was emergently transferred to the hospital where he remained hemodynamically unstable requiring multiple pressors. Due to an acute drop in hematocrit, along with metabolic acidosis, a computed tomography (CT) scan of the abdomen and pelvis was performed. which The scan revealed a large volume of intraperitoneal hemoperitoneum which was largely attributed to CPR. The patient He underwent an exploratory laparotomy during which and the hepatic laceration was repaired and the abdomen was packed. Intraoperatively and postoperatively, the patient remained hemodynamically unstable developing multi-organ failure and requiring multiple products including fresh frozen plasma, packed red blood cells, and cryoprecipitate. Electroencephalogram (EEG) results were consistent with anoxic brain injury and the patient's family opted for comfort care measures only and he expired the next day. Although CPR induced injuries to hepatic structures have scarcely been reported in the literature, this may underestimate their true incidence given the high mortality rate of sudden cardiac arrest requiring CPR. Our case demonstrates that intra-abdominal trauma following CPR, though rare, must needs to always be considered taken into account. and a Importantly, a high index of clinical suspicion is often necessary to make an early diagnosis of liver trauma as a potential life-threatening complication of CPR.
\end{abstract}

Keywords: Hepatic laceration; Cardiopulmonary resuscitation; Bystander; Hemodynamic instability

\section{Introduction}

The overall survival rate for patients who suffer out-of-hospi-

Manuscript accepted for publication February 23, 2017

aDepartment of Trauma and Acute Care Surgery, Forbes Hospital, Allegheny Health Network, Monroeville, PA, USA

${ }^{b}$ Corresponding Author: Saptarshi Biswas, Department of Trauma and Acute Care Surgery, Forbes Hospital, Allegheny Health Network, Monroeville, PA, USA. Email: saptarshibiswas@comcast.net

doi: https://doi.org/10.14740/jmc2775w tal cardiac arrests ranges between $3 \%$ and $10 \%$ [1], with less than $50 \%$ surviving for more than 3 days, and less than $10 \%$ living to be discharged from the hospital [1]. Rapid access to cardiopulmonary resuscitation (CPR), early defibrillation as well as prompt medical care and transfer to an appropriate facility could very possibly help save lives.

A multitude of rare injuries secondary to chest compressions have been reported. Included among these are cases of liver injury attributable to CPR-induced trauma. Interestingly, many of these reported cases cite that CPR was performed by the general bystander outside of a healthcare facility [2], although, notably, injuries have also been reported within healthcare facilities when performed by healthcare professionals [3, 4], and even with the use of an automated CPR device [5, 6].

In the Majority of cases, patients presented with either a low or significantly decreasing hemoglobin and/or hematocrit as early indicators of liver injury [6]. Changes in vital signs and physical exam findings have also been identified as indicators. Reports of abdominal free fluid identified on abdominal ultrasound/focused assessment with sonography for trauma (FAST) have also been described in several cases. Computed tomography (CT) has also been used to describe solid organ injury. Due to the high mortality associated with these injuries and their circumstances, a vast proportion of these cases are only identified at the time of autopsy.

Management of these injuries has been variable due to the differences in severity, circumstances, and comorbidities surrounding them. Volume resuscitation with blood product replacement is commonly performed. Hepatic artery embolization and open surgical repair with packing and general damage control of the injury are other common therapeutic treatments described in the literature.

\section{Case Report}

A 63-year-old male was having lunch with friends in a restaurant when bystanders witnessed him "seize" for approximately one minute. Subsequently, the patient became unresponsive and pulseless. CPR was initially performed by bystanders at the scene, and then by EMS personnel, who achieved return of spontaneous circulation (ROSC) in the field. Unfortunately, this first ROSC was followed by bradycardia and a second arrest. CPR was resumed by the first responders, who also initiated ACLS protocol, and administered additional epinephrine, leading to a second ROSC. The patient presented to the emergency department (ED) unresponsive, apneic, and areflexic with a Glasgow Coma Scale (GCS) score of 3. On arrival to 


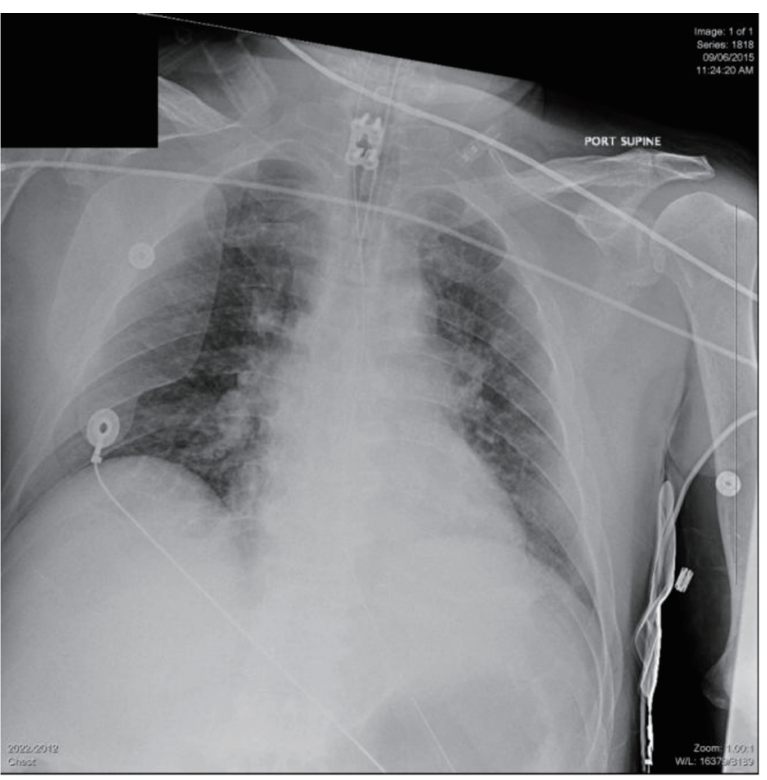

Figure 1. Initial chest X-ray on presentation to the ED.

the ED, he was both hypertensive and tachycardiac.

His past medical history was salient for a transient ischemic attack (TIA) which occurred 11 years prior, and according to the family, had he also suffered a stroke at some time but did not incur any residual disability. His current medications were Plavix and Diovan.

He was emergently intubated in the ED and the endotracheal tube placement was verified by chest X-ray (Fig. 1). His physical examination revealed bilateral fixed, dilated and nonreactive pupil measuring $5 \mathrm{~mm}$. On primary assessment, physical examination revealed bilateral fixed, dilated and nonreactive pupils measuring $5 \mathrm{~mm}$. On cardiovascular exam, heart rate had normalized with regular rhythm and audible S1-S2 without S3 or S4 gallop. He had no jugular venous distention. Pulmonary examination exhibited wheezes and rhonchi. The

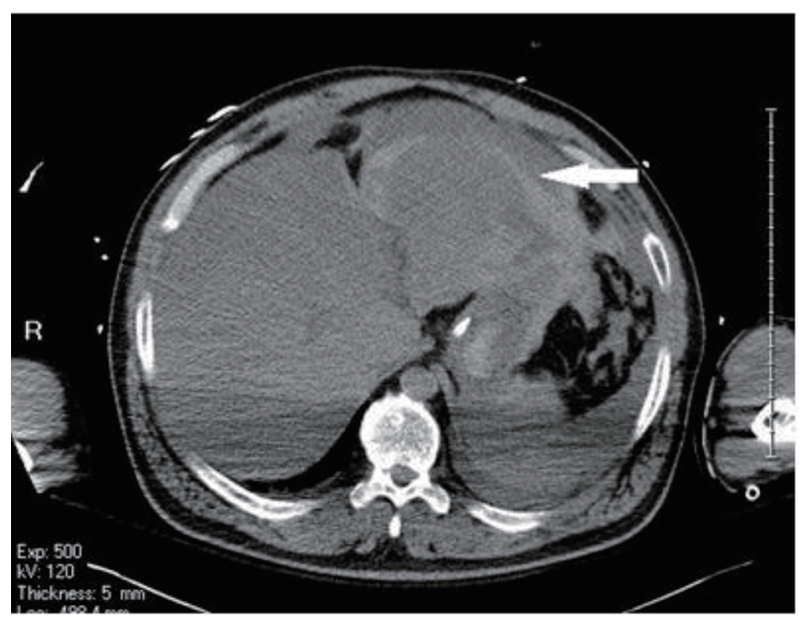

Figure 2. Non-contrast CT scan showing intraperitoneal hemoperitoneum extending from the upper abdomen around the liver and spleen and the stomach.

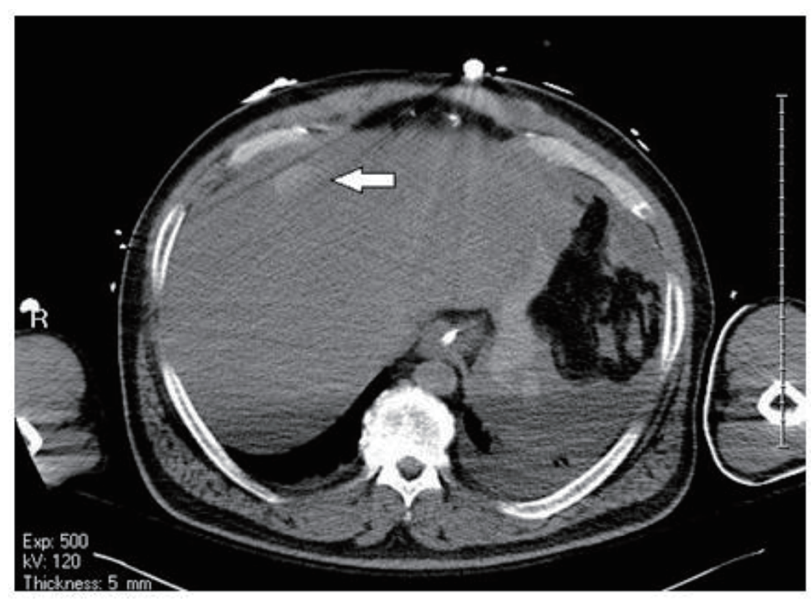

Figure 3. Blush within the liver suggestive of bleed.

abdomen was grossly soft and non-distended. The skin was mottled. Lab results revealed that liver function was markedly abnormal. International normalized ratio (INR) was 1.3. Creatine phosphokinase (CPK) was elevated, although the first troponin was negative. Electrocardiogram (EKG) showed diffuse ST depressions especially in the precordial leads. There was no reciprocal elevation. No previous EKG was available for comparison. CT of the head was negative for any acute intracranial bleed, mass effect, midline shift or extra axial fluid collection. No large vascular territory infarct was identified and the gray white matter differentiation appeared preserved.

The patient was emergently transferred to the intensive care unit (ICU) where he became hypotensive and bradycardic, possibly due to the waning effect of epinephrine. IV epinephrine was therefore given and the patient started on dopamine infusion. Subsequently, he received multiple boluses of crystalloids and colloids, sodium bicarbonate drip, and required multiple pressors, e.g. levophed, epinephrine, and vasopressin infusions to maintain hemodynamics. He was in multiorgan failure. Repeat EKG showed sinus rhythm with small Q waves in the lateral leads as well as $\mathrm{T}$ wave abnormalities in the lateral leads suggestive of lateral wall ischemia or infarction. Cardiology was on board. Considering the hemodynamic instability and neurological status, a decision was made to withhold cardiac catheterization.

In the ICU, a precipitous drop in hemoglobin level from 14 to 6 , as well as marked metabolic acidosis with a lactate of 21 , was noted. Transfusion was started via the Belmont ${ }^{\mathbb{R}}$ Rapid Infuser. Physical exam findings included a tense and significantly distended abdomen.

Acute care surgery was consulted and the patient was sent for a non-contrast $\mathrm{CT}$ scan of the abdomen and pelvis, in the context of acute renal failure, which revealed a large volume of intraperitoneal hemoperitoneum extending from the upper abdomen around the liver and spleen and the stomach through the paracolic gutters into the pelvis (Figs. 2 and 3). No significant retroperitoneal hemorrhage was identified. The etiology of the hemoperitoneum was unclear. No free air was noted to suggest perforation of viscus.

The patient was taken to the operating room for an emergent exploratory laparotomy with a provisional diagnosis of 
hemoperitoneum of unknown etiology, possible abdominal compartment syndrome. The patient was prepped and draped and preoperative broad spectrum antibiotics was administered.

During the exploratory laparotomy, an active bleeding from a $5 \mathrm{~cm}$ laceration, $2-3 \mathrm{~cm}$ deep, was identified immediately overlying the falciform ligament. The bleeding was initially managed with cautery, surgicel, and pressure but with little success. Additional cautery and FloSeal were also ineffective. Hemostasis was achieved after the laceration was closed by two figure-of-eight chromic sutures; however, the wound continued to ooze following another trial of FloSeal and the application of 10 minutes of direct pressure. The bleeding was determined to be most likely due to an underlying coagulopathy. The patient was not pharmacologically anti-coagulated prior to admission but his home medications included Plavix. Platelet count was within normal range on admission; however, at that time, prothrombin time (PT) was $48.8 \mathrm{~s}$ and INR was 5.5. The liver was packed with several laparotomy pads, and a wound vacuum assisted closure (VAC) was applied. During the procedure, the patient received eight units of fresh frozen plasma, six units of packed red blood cells, and one unit of cryoprecipitate.

Despite successfully achieving hemostasis intra-operatively, the patient's condition declined once returned to the ICU. He remained intubated and on pressors following the procedure. Postoperative PT was measured at 31.3 with an INR of 3.1, albumin was decreased at $2.1 \mathrm{~g} / \mathrm{dL}$, and liver enzymes were markedly elevated (ALT 2,880 U/L and AST 2,500 U/L). In the ICU, the patient required additional two units of fresh frozen plasma, two units of packed red blood cells, and one unit of cryoprecipitate postoperatively.

It became clear that the patient's decline was attributable in large part to anoxic visceral injuries incurred during the initial arrest. He remained hypotensive with evidence of shock liver and multi-organ system failure. Repeat electroencephalogram (EEG) results were consistent with anoxic brain injury, and fairly monotonous theta waves were concerning for progression towards theta coma, which is usually a poor prognosis in the setting of cardiac arrest. The patient's family opted for comfort care measures only, palliative care was consulted and he expired the next day.

\section{Discussion}

Thoracic trauma is a relatively well-known complication, and includes rib fractures, sternal fractures, chest wall hematoma, pneumothorax, hemothorax, hemopericardium and cardiac contusion $[7,8]$. Sternal fractures are reported to occur in $1 / 5$ resuscitation attempts, while costal fractures occur in $1 / 3$; over $65 \%$ of patients with costal fractures had three or more fractures in the adjacent ribs [9].

In a prospective study of cardiac resuscitation complication authored by Krischer et al. [10], 705 cases were autopsied to identify the cause of death and the pathological findings attributable to CPR, of which $31.6 \%$ had rib fractures, $21.1 \%$ had sternal fractures while $18.3 \%$ had anterior mediastinal complications. Interestingly, in this study, 30.8\% reported abdominal visceral complications with liver rupture of $2.1 \%$, while other liver injuries were $0.8 \%$. Comparatively pulmonary complication accounted for $13 \%$. Heart and great vessel injuries were significantly rarer at less than $0.5 \%$. Meron et al mentioned CPR-induced hepatic injuries in only $0.6 \%$ of cases [6], while Clark et al reported a frequency of $21 \%$ [11].

Although isolated cases of liver injury secondary to chest compressions appear in the literature, this complication of CPR remains extremely rare. A retrospective study was conducted at the General Hospital of Vienna in which only 15 cases of liver injury (from a pool of over 2,500 cardiac arrest patients) were found over a 14-year period [6].

Hepatic, splenic along with intestinal injuries are the commonest of the rarely reported intra-abdominal complications of CPR [12]. Hepatic injury is the commonest of the CPR-related intra-abdominal complication with an approximate incidence of $0.6 \%[5,6,10,13-16]$. Splenic rupture ranks second [6]. Intraperitoneal bleeding and retroperitoneal hematoma were also reported.

The left hepatic lobe is most frequently injured $[6,10,12]$. This is often attributed to the close anatomical proximity of the left lobe of the liver and the xiphoid process of the sternum especially in cases of improper hand placement for performing chest compressions [12]. However, there are instances of injury to the right lobe as well $[6,17]$. Pollak et al in an autopsy series showed that the most common CPR-related liver lacerations occur on the medial line [18]. Several authors have questioned whether the method of chest compressions, in terms of who (or what, in the case of an automatic device) performs them and the technique used, have any bearing on the incidence of liver (and other intra-abdominal) injury. Some experts have opined that exertion of increased pressure during chest compression can lead to crush injury to the underlying viscera [19]. There are also reports of intra-abdominal complications even with light compressions with a correct hand position [20, 21].

Prolonged compressions have not been shown to cause a higher incidence of liver injury [3]. It is also important to reiterate that patients have sustained these injuries even when trained emergency responders, including emergency physicians performed the compressions [4]. Therefore, due to the fact that compressions, though possibly prolonged, were performed both by family members (lay persons) and professionally trained emergency responders, this case does not offer additional evidence to support any particular theory regarding the etiology of these injuries.

Some authors have cited hepatic ischemia and hepatic congestion secondary to venous congestion as the potential aggravating risk factors for liver rupture [12]. Antiplatelet, antithrombotic and thrombolytic treatments especially in patients with myocardial ischemia have also been cited as precipitating factors $[1,14,15]$.

Focused attention on cardiac condition following arrest may often distract the need for further workup. Several signs and symptoms of hemorrhage are also shared with impaired cardiac function, such as hemodynamic instability. For these reasons, intra-abdominal hemorrhage and liver injury are clinical developments that may either be completely missed or diagnosed belatedly. Therefore, incidence may be underreported and patients would likely benefit from increased surveillance.

As intra-abdominal complications of CPR are relatively rare, the diagnosis at the outset can be difficult and usually requires thorough clinical evaluation, high degree of suspicion, regular careful 
hemodynamic monitoring along with laboratory parameters [4]. In our case the intra-abdominal bleed was initially suspected due to persistent hypotension requiring significant pressors, abdominal distention and a continuing downtrending hematocrit.

When a hepatic rupture is suggested, ultrasound may be used as the initial step in imaging studies. FAST exam is easily performed in the ED and in the ICU setting. It is safe, requires much less time than CT scan, and shows presence of peritoneal fluid int he right upper quadrant. It is widely considered as the best initial imaging method especially for hemodynamically unstable patients [22] The limitations include being operatordependent, and sometimes failing to detect fluid volumes less than $200-500 \mathrm{~mL}$ [23].

CT scan is currently the imaging tool of choice and detects hepatic laceration or rupture. It has a high sensitivity and specificity in detecting solid organ injury [24, 25]. CT also helps with staging and deciding if surgery is necessary. Hepatic injury like most of other organs is graded using the standards for Organ Injury Scaling Committee for the American Association of the Surgery of Trauma (AAST).

Lower-grade injuries are typically managed conservatively [26], while higher-grade injuries often require angioembolization or surgery. There are case reports [4] of emergent angiography and blind embolization of the right and middle hepatic artery when no definite bleeding source could be identified. However, clinical circumstances should cover decisionmaking in the management of these patients.

Of course, not all cases have been managed with an open surgical procedure as was the case with our patient. Some authors have suggested that conservative management [27] with hepatic arterial embolization together with replacement of blood products may be preferred over laparotomy due to a perceived high mortality rate associated with surgical intervention [2].

Naess et al [2] made the assumption of a cause and effect association between surgical intervention and the mortality of patients with these types of injuries. This may not be an absolutely correct assumption as the causes of death for the majority of patients described in the literature (both those managed surgically and conservatively) have not been always attributed to bleeding, but rather to the original cardiac arrest or other compounding factors and conditions secondary to the arrest or hospitalization [6].

The importance of coagulopathy cannot be overstated in the discussion of liver injury caused by CPR. The coagulopathy identified intraoperatively in our patient could have contributed to the hepatic hemorrhage. Trauma-induced coagulopathy is well recognized. It is a hypocoagulable condition usually occurring within the first day following major injury. Tissue hypoperfusion resulting in protein $\mathrm{C}$ activation, hyperfibrinolysis, clotting factor dysfunction and endothelial glycocalyx degradation have all been proposed as possible mechanisms. Acidosis, hemodilution and hypothermia during resuscitation aggravate ongoing coagulopathy. It has been noted in the literature that therapeutic hypothermia (induced with a goal of improving neurologic function) has a strong association with the development of coagulopathy [3]. Hypothermia may have played a role in this coagulation as the patient had to be warmed intra-operatively due to a body temperature drop to $33^{\circ} \mathrm{C}$. The hypothermia in this patient was initially therapeuti- cally induced and aggravated by the abdominal cavity being open for the duration of the emergency laparotomy. That is to say, the blood loss in this patient was not believed to have precipitated the anoxic brain injury. It was well controlled and losses were managed appropriately, thus precluding it as an aggravating factor in the patient's eventual demise. Still, we believe it is appropriate to intensify efforts to maintain homeostatic body temperature during exploratory laparotomy in order to prevent the exacerbation of any underlying coagulopathy which may lead to increased blood losses.

Thromboelastography and ROTEM can potentially recognize early trauma-induced coagulopathy and guide in transfusion of blood products. Such viscoelastic assay-guided resuscitation in trauma patients requiring massive transfusion has been shown in literature to reduce mortality. In addition, it may also be prudent to draw coagulation labs (including PT, aPTT and INR) as well as a complete blood count, hemoglobin and hematocrit immediately upon presentation for any patient presenting with blunt trauma to the thorax or abdomen, including patients who are status post chest compressions. This may provide guidance regarding the need for further diagnostic workup for intra-abdominal injury and give the surgical team baseline knowledge of any coagulopathy which could complicate emergent surgical procedures.

Finally, several authors have questioned whether the method of chest compressions, in terms of who (or what, in the case of an automatic device) performs them and the technique used, have any bearing on the incidence of liver (and other intra-abdominal) injury. It may well be that direct pressure to the xiphoid process during compressions is frequently responsible for these injuries [6], especially considering that several of the reported injuries have involved the anterior left hepatic lobe [5], but this has not been conclusively proven.

Prolonged compressions have also not been shown to cause a higher incidence of liver injury [3]. It is also important to reiterate that patients have sustained these injuries even when trained emergency responders, including emergency physicians performed the compressions [4]. Therefore, due to the fact that compressions, though possibly prolonged, were performed both by family members (lay persons) and professionally trained emergency responders, this case does not offer additional evidence to support any particular theory regarding the etiology of these injuries.

\section{Conclusion}

Future studies should focus on the most common underlying causes of liver injury secondary to compressions. Attention should be paid to appropriate hand positioning and/or force exerted during compressions, as well as to preexisting coagulopathy. This would provide further evidence as to whether these injuries are more often sustained by under trained caregivers or if injuries are unavoidable.

With the aim of avoiding a missed diagnosis, studies could include diagnostic imaging of the xiphoid process and early lab testing of coagulation, hemoglobin and hematocrit as part of a protocol of post-CPR management. This could have the benefit of improving patient care while also providing information to help avoid causing these injuries in the future. 


\section{Consent}

Written consent could not be obtained from the deceased patient. However, every effort has been made to protect the identity of the patient.

\section{Competing Interests}

The authors declare that they have no competing interests.

\section{References}

1. Mullany DV, Ziegenfuss MD. Traumatic liver injury complicating cardiopulmonary resuscitation. The value of a major intensive care facility: a report of two cases. Critical Care and Resuscitation. 2004;6(2):102-104.

2. Naess PA, Engeseth K, Grotta O, Andersen GO, Gaarder C. Minimal invasive treatment of life-threatening bleeding caused by cardiopulmonary resuscitation-associated liver injury: a case report. J Med Case Rep. 2016;10(1):132.

3. Zahn G, Hauck M, Pearson DA, Green JM, Heffner AC. Major hemorrhage from hepatic laceration after cardiopulmonary resuscitation. Am J Emerg Med. 2015;33(7):991 e993-994.

4. Jin Wi, Dongho Shin. Liver Laceration with Hemoperitoneum after Cardiopulmonary Resuscitation. Korean Journal of Critical Care Medicine. 2014;29(2): 141-143.

5. Camden JR, Carucci LR. Liver injury diagnosed on computed tomography after use of an automated cardiopulmonary resuscitation device. Emerg Radiol. 2011;18(5):429431.

6. Meron G, Kurkciyan I, Sterz F, Susani M, Domanovits $\mathrm{H}$, Tobler K, Bohdjalian A, et al. Cardiopulmonary resuscitation-associated major liver injury. Resuscitation. 2007;75(3):445-453.

7. Jain V, Lutchmansingh D, Kaur D, Kaul P, Trikha G, Abbasi A. Liver laceration as a complication of cardiopulmonary resuscitation. Am J Respir Crit Care Med. 2014;189.

8. Buschmann CT, Tsokos M. Frequent and rare complications of resuscitation attempts. Intensive Care Med. 2009;35(3):397-404.

9. Kottachchi DT, Dong J, Reid S. A rare complication of cardiopulmonary resuscitation. Can J Surg. 2009;52(1):E12.

10. Krischer JP, Fine EG, Davis JH, Nagel EL. Complications of cardiac resuscitation. Chest. 1987;92(2):287-291.

11. Clark DT. Complications following closed-chest cardiac massage. JAMA. 1962;181:337-338.

12. Kaplon-Cieslicka A, Kosior DA, Grabowski M, Rdzanek A, Huczek Z, Opolski G. Coronary artery dissection, traumatic liver and spleen injury after cardiopulmonary resuscitation - a case report and review of the literature. Arch Med Sci. 2013;9(6):1158-1161.

13. Rosen J, Tuchek JM, Hartmann JR. Liver laceration in the hemodynamically unstable post-cardiac massage pa- tient: early recognition and management - case report. J Trauma. 1999;47(2):408-409.

14. Adams HA, Schmitz CS, Block G, Schlichting C. [Intraabdominal bleeding after myocardial infarction with cardiopulmonary resuscitation and thrombolytic therapy]. Anaesthesist. 1995;44(8):585-589.

15. Pezzi A, Pasetti G, Lombardi F, Fiorentini C, Iapichino G. Liver rupture after cardiopulmonary resuscitation (CPR) and thrombolysis. Intensive Care Med. 1999;25(9):1032.

16. Druwe PM, Cools FJ, De Raedt HJ, Bossaert LL. Liver rupture after cardiopulmonary resuscitation in a patient receiving thrombolytic therapy. Resuscitation. 1996;32(3):213-216.

17. Kouzu H, Hase M, Kokubu N, Nishida J, Kawamukai M, Usami Y, Hirokawa N, et al. Delayed visceral bleeding from liver injury after cardiopulmonary resuscitation. J Emerg Med. 2012;43(4):e245-248.

18. Pollak S, Reiter C, Stellwag-Carion C. [2-stage rupture of the liver as a complication of external heart massage]. Z Rechtsmed. 1984;92(1):67-75.

19. Umach $\mathrm{P}$, Unterdorfer $\mathrm{H}$. [Massive organ injuries resulting from resuscitation measures]. Beitr Gerichtl Med. 1980;38:29-32.

20. Smekal D, Johansson J, Huzevka T, Rubertsson S. No difference in autopsy detected injuries in cardiac arrest patients treated with manual chest compressions compared with mechanical compressions with the LUCAS device - a pilot study. Resuscitation. 2009;80(10):1104-1107.

21. Gil Martin FJ, Perez Ordonez A, Castelo Tarrio I, Morentin Campillo B. Liver laceration caused by cardiopulmonary resuscitation manoeuvres. Emergencias. 2009;21(2):148-150.

22. Korner M, Krotz MM, Degenhart C, Pfeifer KJ, Reiser MF, Linsenmaier U. Current Role of Emergency US in Patients with Major Trauma. Radiographics. 2008;28(1):225-242.

23. Sheng AY, Dalziel P, Liteplo AS, Fagenholz P, Noble VE. Focused Assessment with Sonography in Trauma and Abdominal Computed Tomography Utilization in Adult Trauma Patients: Trends over the Last Decade. Emerg Med Int. 2013;2013:678380.

24. Yoon W, Jeong YY, Kim JK, Seo JJ, Lim HS, Shin SS, $\mathrm{Kim} \mathrm{JC}$, et al. CT in blunt liver trauma. Radiographics. 2005;25(1):87-104.

25. Becker CD, Mentha G, Terrier F. Blunt abdominal trauma in adults: role of $\mathrm{CT}$ in the diagnosis and management of visceral injuries. Part 1: liver and spleen. Eur Radiol. 1998;8(4):553-562.

26. Eastern Association for the Surgery of Trauma (EAST) Practice Management Guidelines Work Group. Practice Management Guidelines for the Nonoperative Management of Blunt Injury to the Liver and Spleen. 2003. www. east.org/tpg/livspleen.pdf.

27. Stassen NA, Bhullar I, Cheng JD, Crandall M, Friese R, Guillamondegui O, Jawa R, et al. Nonoperative management of blunt hepatic injury: an Eastern Association for the Surgery of Trauma practice management guideline. J Trauma Acute Care Surg. 2012;73(5 Suppl 4):S288-293. 